\title{
LAS REDES SOCIALES INFLUYEN EN EL COMPORTAMIENTO DE LOS UNIVERSITARIOS
}

Jabalera Sierra, Paula Mํㅗㄴ (paula7@correo.ugr.es)

Morey Amer, Mํㅡㄹ del Mar (marmorey88@correo.ugr.es)

Rodríguez Bonachera, Antonio (delate@correo.ugr.es)

Sánchez Molina, Ana Belén (anabsm@correo.ugr.es)

Universidad de Granada

Fecha de recepción: 28 de mayo de 2012

Fecha de aceptación: 11 de junio de 2012

Localizador: http:www.ugr.es/local/miguelgr/ReiDoCrea-Vol.1-Art.12-Jabalera-Monrey-Sanchez.pdf

RESUMEN: Las redes sociales son un medio muy utilizado hoy en día por el colectivo universitario, de este modo nos centramos en investigar su influencia en el comportamiento de éstos, para comprobar hasta qué punto se dejan de lado las relaciones interpersonales por la utilización de éstas, así como las actividades más utilizadas en las mismas, y las que se dejan de realizar por su utilización. Nos centramos en un colectivo de la universidad de Granada.

PALABRAS CLAVE: Redes sociales, relaciones interpersonales, comunicación, influencia social, disonancia cognitiva.

ABSTRACT: Social networks are a tool which is used considerably today by university students. For this reason, we will focus on investigating their influence on the behavior of the students, to determine to what extent they leave their interpersonal relationships and their activities to be connected with social networks. We will also research which activities on the social networks are the most used by the students. We focus on a group of students from the University of Granada.

KEYWORDS: Social network, interpersonal relationships, communication, social influence, cognitive dissonance. 


\section{INTRODUCCIÓN}

Hoy en día las redes sociales se han convertido en uno de los principales medios de comunicación e información entre los universitarios de nuestra sociedad, de esta forma se mantienen informados de lo que ocurre a su alrededor y entretenidos, ya que se han convertido en una gran fuente de ocio y de poder. Generalmente, se consideran como "servicios basados en la web que permiten a sus usuarios relacionarse, compartir información, coordinar acciones y en general, mantenerse en contacto" (Orihuela, 2008, p.2).

Esta comunicación virtual se realiza con el fin de comunicarse de una forma instantánea y rápida entre personas distanciadas, prefiriendo la pantalla y el teclado, haciendo que las relaciones interpersonales se vean deterioradas, ya que dichas relaciones consisten en la comunicación y contacto físico entre personas, con el fin de obtener información en relación con el entorno al que pertenecen. Según Cachia (2008) es más fácil contactar con diferentes personas sin ser necesario establecer una relación muy afianzada, se crean unas relaciones más débiles, en las que no existe una conexión emocional pero si un intercambio de información, es decir se crean unas conexiones mas sueltas y menos comprometidas en las que no hace falta mucho tiempo y esfuerzo.

El proceso comunicativo está constituido por un conjunto de señales emitidas (sonidos, gestos, señas) con la finalidad de dar a conocer un mensaje. Para que exista una comunicación adecuada debe de estar compuesta por un receptor capacitado para codificar un mensaje e interpretarlo, de tal forma que si la comunicación falla, difícilmente se llevará a cabo una relación interpersonal. Lo que se ve más afectado en dichas relaciones es el contacto físico (relaciones entre personas tú a tú), además el medio y el entorno. En este sentido la relación y comunicación entre personas en las redes sociales es diferente, porque se pueden seleccionar con más facilidad las personas a las que queremos dirigirnos e ignorar a las que nos incomodan o no tenemos interés, es decir, "los individuos están dispuestos a contar como amigos en las redes sociales con todos aquellos que no les disgusta" (Gross y Acquisti, 2005, p.3).

Teniendo en cuenta estos aspectos, según Nicholas, A. Chritakis y James H. Flowler han informado que la influencia y el impacto que ejercen, en la sociedad, las redes sociales llegan a una potencialidad capaz de transformar a ésta y a las organizaciones, haciendo que superemos la individualidad para conseguir aquello de los que somas capaces de logar por nosotros mismos. Esta influencia se ve reflejada en el comportamiento de las personas, entre otras cosas, por lo contactos o "amigos" que se tienen, ya que esta influencia que ejerce nuestro entorno sobre nosotros, nos lleva a comportarnos de un modo porque alguien se comporta de ese modo y las redes sociales amplifican esos horizontes haciendo de los considerado correcto un epidemia. Siguiendo la línea de estos mismo autores exponen " las redes sociales difunden felicidad, generosidad y amor. Siempre están ahí ejerciendo una influencia sutil y al mismo tiempo determinante en nuestras elecciones, acciones, pensamientos y sentimientos y también nuestros deseos. Además, esas conexiones no terminan en las personas que conocemos. Mas allá de nuestros horizontes sociales, los amigos de los amigos de nuestros amigos pueden impulsar reacciones en cadena que acaben por alcanzarnos, como olas que, venidas desde tierras ajenas, rompen en nuestras playas".

Tal importancia ha obtenido las redes sociales en nuestra población universitaria que de ser una diversión en el tiempo libre ha pasado a formar parte de una obligación, es una necesidad para poder estar en contacto con personas conocidas. Si no estamos 
dentro del espacio virtual hoy en día falta una parte de la vida social, una vida que está surgiendo entre el mundo físico y mundo virtual, en el que nos informamos sobre la vidas ajenas, nos comunicamos y nos exponemos tal y como queremos. Sirven para afianzarse dentro de un grupo sentirse socialmente aceptado que es un punto fundamental del desarrollo humano, y hace que en muchas ocasiones se discrimina a las personas que no utilizan esta forma para comunicarse.

Como hemos podido ver la comunicación a través de redes sociales no es perjudicial hasta el momento que aparece el conflicto o la disonancia cognitiva, cuando dejamos de lado las relaciones cercanas con los amigos para centrarnos en las relaciones virtuales y no saber elegir entre ambas, decantándonos por la opción que hoy en día es más atractiva, por tanto las redes sociales.

\section{MATERIAL Y MÉTODOS}

A partir de aquí planteamos el problema de nuestra investigación que es comprobar que el comportamiento de las personas se ve influenciado por el uso excesivo de las redes sociales, siendo éste nuestro principal objetivo, del que sacamos los siguientes subobjetivos:

Conocer a cantidad de tiempo utilizado en indagar en las redes sociales.

Comprobar qué actividades, tanto lúdicas como académicas, se llevan a cabo, con más frecuencia, en las redes sociales.

Conocer con qué frecuencia abandonan ciertas actividades cotidianas por la utilización de las redes sociales.

En cuanto a las variables implicadas en esta investigación, encontramos como variable independiente la influencia de las redes sociales, y como variable dependiente el comportamiento de los jóvenes universitarios. Además, debemos tener en cuenta en la realización de la investigación, la existencia de abundantes variables extrañas, como pueden ser el estado de ánimo, el momento y lugar en el que se realizó el cuestionario, etc.

Para nuestra investigación hemos utilizado un diseño metodológico cualitativo. El método fundamental en este trabajo ha sido el cuestionario. Referente a la población de estudio, ésta ha sido constituida por estudiantes universitarios de la Facultad de Ciencias de la Educación, de la Universidad de Granada (200 alumnos de los cuales, 10 no contestaron, por tanto nos centraremos en el $95 \%$, que corresponde con la muestra real).

No nos hemos centrado en la edad y el sexo de la muestra, puesto que no encontramos discrepancia entre sexos a la hora de utilizar las redes sociales, y en cuanto a la edad, al centrarnos en el colectivo universitario sabemos que partimos de la edad de 18 años en adelante, pero no nos centramos en la influencia en cuanto a la edad sino en el comportamiento general de un/a universitario.

Se diseñó un cuestionario específico para la investigación, adaptando las preguntas del mismo a los objetivos de esta investigación. Este cuestionario está compuesto por 47 ítems, donde se debe responder mediante escala LIKERT de 5 puntuaciones $(1=$ nada, $5=$ mucho). Con la contabilización de estas respuestas obtuvimos los promedios y desviaciones.

Las preguntas exploraban la cantidad de tiempo utilizado en las redes sociales, la cantidad de tiempo dedicado, dentro de éstas, a la realización de diversas actividades 
(mensajes, chat, fotos, etc.); a la cantidad de tiempo que se abandonan otras actividades cotidianas por la utilización de las redes sociales y la frecuencia en que se utilizan las redes sociales en las actividades académicas.

La investigación se llevó a cabo durante la última semana de abril y las dos primeras semanas de mayo del 2012. Una vez obtenidos los datos, se generó una base de datos, analizada con el programa SPSS.

\section{RESULTADOS}

Una vez analizados los datos, obtenemos que la muestra total es un 95\%, del cual, $90,3 \%$ respondió que utilizaban las redes sociales y el 4,7\% no las utilizaban. De este modo los datos y porcentajes que exponemos a partir de ahora se han calculado a partir de esta base (90,3\%).

El $61,3 \%$ de los encuestados respondieron que se conectan varias veces al día a las redes sociales, de las cuales las más utilizadas son Facebook $88,9 \%$, Twitter $43 \%$ y Tuenti $74 \%$. Por lo que dichas redes ha llegado a formar parte de la vida cotidiana de nuestros encuestados $(M=2,81$; D.T=1,09) como se puede observar hay una media relativamente alta.

Llegados a este punto, pasamos a analizar e interpretar los resultados relacionándolos con los objetivos establecidos anteriormente, en cuanto al primer objetivo, se ven reflejados los resultados en la tabla 1.

\begin{tabular}{|l|l|l|l|}
\hline Tabla 1. Cantidad de tiempo dedicado a cada herramienta \\
\hline & Porcentaje & Media & Desv. Tip. \\
\hline Privados/mensajes & $63,47 \%$ & 3,17 & 1,01 \\
\hline Fotos & $63,05 \%$ & 3,15 & 1,10 \\
\hline Chat & $69,47 \%$ & 3,47 & 1,39 \\
\hline Muro/noticias & $57,89 \%$ & 2,89 & 1,10 \\
\hline Vídeo & $44,73 \%$ & 2,23 & 1,11 \\
\hline Eventos & $43,36 \%$ & 2,17 & 1,08 \\
\hline Reproducir música & $67,05 \%$ & 3,35 & 1,34 \\
\hline Notas & $46,63 \%$ & 2,33 & 1,12 \\
\hline Enlaces de contenido & $48,42 \%$ & 2,42 & 1,12 \\
\hline Grupos & $50,10 \%$ & 2,50 & 1,19 \\
\hline Juegos & $42,84 \%$ & 2,14 & 1,29 \\
\hline
\end{tabular}

Observando la recopilación de los resultados con respecto a la cantidad de tiempo dedicado a cada herramienta vemos que el medio más utilizado es el chat con un porcentaje de $69,47 \%$, con una media de 3,47 y una DT de 1,10. Y el menos utilizado son los juegos son un porcentaje de un 42,84 \%, una media de 2,14 y una DT de 1.29 quedando el resto de las opciones con valores intermedios entre el chat y juegos.

El chat es un medio rápido e instantáneo que permite la comunicación de las personas tanto lejanas como cercanas, además de poder intercambiar todo tipo de información y documentos, por esta razón es el medio más utilizado dentro de las redes sociales. 
En cuanto al segundo objetivo, los resultados se ven reflejados en la tabla 2.

Tabla 2. Cantidad de tiempo dedicado en las redes sociales a distintas actividades:

\begin{tabular}{|c|c|c|c|}
\hline & Porcentaje & Media & Desv. Típ. \\
\hline Quedar con mi grupo de amigos & $72 ' 10 \%$ & $3{ }^{\prime} 60$ & 1 '12 \\
\hline Informarme sobre lo que pasa en mi grupo de amigos & $71 ’ 05 \%$ & $3 ’ 55$ & $1 ’ 07$ \\
\hline Comentar fotos/videos/otros comentarios & $62 ' 84 \%$ & $3{ }^{\prime} 14$ & 1 1'07 \\
\hline Compartir información, archivos, fotos, documentos & $6273 \%$ & $3 ’ 14$ & $1 ’ 07$ \\
\hline Cotillear/curiosear & $62 ' 52 \%$ & 3,13 & 1 '24 \\
\hline Retomar el contacto con otras personas & $63 ’ 57 \%$ & $3{ }^{\prime} 18$ & 1 '04 \\
\hline Informarme sobre la actualidad (noticias) & $63{ }^{\prime} 47 \%$ & $3 \prime 19$ & $1 ’ 07$ \\
\hline Contar lo que estoy haciendo & $45 ’ 89 \%$ & $2 ' 29$ & 1 '14 \\
\hline Hacer amigos & $4673 \%$ & 234 & $1 ' 22$ \\
\hline Usar las aplicaciones y juegos de la RS & $40 ' 10 \%$ & $2{ }^{\prime} 00$ & $1 ' 20$ \\
\hline Compartir mis sentimientos & $41^{\prime} 47 \%$ & $2{ }^{\prime} 07$ & 1 1'09 \\
\hline Hacer nuevos contactos a nivel profesional & $45^{\prime} 89 \%$ & 229 & 1 '19 \\
\hline Buscar pareja/ligar & $33 ’ 05 \%$ & 1 1'65 & 1 '08 \\
\hline
\end{tabular}

Con respecto a la cantidad de tiempo dedicado en las redes sociales a distintas actividades vemos que la actividad más recurrente es la de quedar con el grupo de amigos, con un porcentaje de $72 ' 10 \%$ (M= 3'60 y DT=1'12). Por otro lado, la actividad a la que menos tiempo se dedica es la del uso de aplicaciones y juegos, con un porcentaje de 40'10 (M= 2'00 y DT= 1'20). Por lo que se da a entender que por lo que más se utilizan las redes sociales es para establecer relaciones interpersonales, cercanas, ya que sirve de comunicación para encontrarse el grupo de amigos posteriormente. Por tanto, vemos que las relaciones interpersonales no están tan influenciadas como pensábamos antes de la realización de la investigación.

En cuanto al tercer objetivo, los resultados obtenidos se ven reflejados en la tabla 3.

\begin{tabular}{|c|c|c|c|}
\hline & Porcentaje & Media & Desv. Típ. \\
\hline Ver la televisión & $56 ' 73 \%$ & $2 ' 84$ & $1 ' 23$ \\
\hline Estar sin hacer nada & $49^{\prime} 47 \%$ & $2 ' 47$ & $1 ' 21$ \\
\hline Estudiar & $56^{\prime} 10 \%$ & $2{ }^{\prime} 80$ & 1 '05 \\
\hline Leer & 53 '26\% & 2 '68 & 1 '17 \\
\hline Dormir & $58 \%$ & $2 ' 9$ & 1'31 \\
\hline Escuchar la radio & $50,10 \%$ & $2 ' 50$ & $1 ' 22$ \\
\hline Hacer deporte & $50 ' 73 \%$ & $2 ' 54$ & $1 ' 25$ \\
\hline
\end{tabular}




\begin{tabular}{|l|l|l|l|}
\hline Ir al cine & $47^{\prime} 47 \%$ & $2^{\prime} 37$ & $1^{\prime} 20$ \\
\hline Pasear/estar con amigos-familia & $566^{\prime} 42 \%$ & $2^{\prime} 82$ & $11^{\prime 26}$ \\
\hline Trabajar & $50 \%$ & $2^{\prime} 5$ & $1^{\prime} 29$ \\
\hline Otras actividades & $53^{\prime} 05 \%$ & $2^{\prime} 65$ & $1^{\prime} 23$ \\
\hline
\end{tabular}

Teniendo en cuenta las actividades a las que se dedican menos tiempo desde que utilizan las redes sociales, el ir al cine $47,47 \%\left(M=2{ }^{\prime} 37\right.$; DT $\left.=1{ }^{\prime} 20\right)$ es a la que menos tiempo dedican, dejando por otro lado, el mayor tiempo a dormir $58 \%$ (M=2'9; DT= 1'31) puesto que es una necesidad vital, aún así, vemos que se dejan mucho tiempo a realizar otras actividades como puede ser ver la televisión $56{ }^{\prime} 73 \%$ (M= 2'84; DT= $1,23)$, o pasear/estar con amigos-familias $56{ }^{\prime} 42 \%\left(M=2{ }^{\prime} 82\right.$; $\left.D T=1^{\prime} 26\right)$ por tanto se sigue manteniendo que no se ven afectadas las relaciones interpersonales por la utilización de las redes sociales.

En cuanto al cuarto y último objetivo, podemos ver los resultados reflejados en la tabla 4.

\begin{tabular}{|c|c|c|c|}
\hline & Porcentaje & Media & Desv. Típ. \\
\hline $\begin{array}{l}\text { Solucionar dudas de los contenidos o exámenes con otros } \\
\text { estudiantes. }\end{array}$ & $71^{\prime} 89 \%$ & $3{ }^{\prime} 59$ & 1 '07 \\
\hline Para saber qué se ha hecho en clase cuando no he asistido & $74^{\prime} 89 \%$ & $33^{\prime} 74$ & 1 '05 \\
\hline Para hacer trabajos de clase & $70,52 \%$ & 3 '53 & 1 '13 \\
\hline $\begin{array}{l}\text { Para estar al día de lo que ocurre en la asignatura (cambios, } \\
\text { imprevistos) }\end{array}$ & $74^{\prime} 63 \%$ & 373 & $1^{\prime} 06$ \\
\hline Para intercambiar apuntes de clase & $633^{\prime} 05 \%$ & $3 \prime 15$ & $1 ' 17$ \\
\hline $\begin{array}{l}\text { Para intercambiar documentación y recursos útiles para la } \\
\text { asignatura }\end{array}$ & $666^{\prime} 36 \%$ & $33^{\prime} 39$ & 1 '09 \\
\hline Resolver dudas sobre mi vida en la universidad & $61^{\prime} 78 \%$ & 3 '09 & 1 '12 \\
\hline Informarse de actividades que organiza la universidad & $577^{\prime} 68 \%$ & $2{ }^{\prime} 88$ & $1 ' 13$ \\
\hline Organizar actividades extra académicas & $53 ’ 57 \%$ & $2{ }^{\prime} 68$ & 1 '25 \\
\hline $\begin{array}{l}\text { Para consultar recomendaciones de libros o recursos que hace el } \\
\text { profesor }\end{array}$ & $533^{\prime} 36 \%$ & $2{ }^{\prime} 67$ & $1^{\prime} 22$ \\
\hline Para contactar con expertos de los temas de estudio & $48,52 \%$ & $2{ }^{\prime} 43$ & $1 ' 19$ \\
\hline Tutorías, consultas al profesor & $56{ }^{\prime} 84 \%$ & 2 '84 & $1 ' 23$ \\
\hline
\end{tabular}

Finalmente, la frecuencia semanal con la que usan las redes sociales para diferentes actividades académicas vemos que la más utilizada es para saber que se ha hecho en clase cuando no han asistido $74^{\prime} 89 \%$ (M= 3'74; DT= 1'05), por tanto, nos da a entender que se utiliza para estar en contacto con la asignatura, y a la vez interactuar con compañeros. Por otra parte, la menos utilizada es contactar con expertos de los temas de estudio 48'52\% (M= 2'43; DT= 1'19), por tanto suponemos, que se utilizan otros medios a la hora de contactar con ellos. 


\section{DISCUSIÓN Y CONCLUSIONES}

Como conclusión decir que teniendo en cuenta los resultados obtenidos, las redes sociales influyen en el comportamiento de los universitarios, ya que éstos pasan un gran período de tiempo al día "conectados", sin embargo, no se dejan de lado las relaciones interpersonales cercanas, ya que un gran porcentaje las utilizan para contactar con las personas que tengan, cerca o lejos, bien para establecer relaciones online o bien para, posteriormente, mantenerlas en persona, es decir, se utilizan como medio de comunicación. Por otro lado, se utilizan con gran frecuencia en las relaciones académicas.

\section{REFERENCIAS BIBLIOGRÁFICAS}

Cachia, R. (2008): Los sitios de creación de redes. Telos: Cuadernos de comunicación e innovación, 76, $69-84$.

Gross, R. y Acquisti, A. (2005): Information Revelation and Privacy in online social Network. Prestado en Workshop on Privacy in the Electronic Society. Alexandria: ACM press.

Nicholas, A., Christakis y James H. Fowler (2010): Conectados: El sorprendente poder de las redes socials y cómo nos afectan. Taurus.

Orihuela, J. L. (2008):Internet: la hora de las redes sociales. Nueva Revista de Política, Cultura y Arte, 119, $57-62$. 